\title{
Rehabilitation Nursing Care of a Child with Congenital Heart Disease with Cerebral Infarction After Valve Replacement: A Case Report
}

\author{
Yuena Zeng ${ }^{1,2}$, Fengxia Yan ${ }^{1, *}$ \\ ${ }^{1}$ School of Nursing, Jinan University, Guangzhou, China \\ ${ }^{2}$ Department of Rehabilitation, the First Affiliated Hospital of Jinan University, Guangzhou, China \\ Email address: \\ yanfengxia0807@163.com (Fengxia Yan) \\ ${ }^{*}$ Corresponding author
}

To cite this article:

Yuena Zeng, Fengxia Yan. Rehabilitation Nursing Care of a Child with Congenital Heart Disease with Cerebral Infarction After Valve Replacement: A Case Report. Rehabilitation Science. Vol. 6, No. 3, 2021, pp. 49-52. doi: 10.11648/j.rs.20210603.12

Received: September 11, 2021; Accepted: September 27, 2021; Published: September 29, 2021

\begin{abstract}
Background: Cerebral infarction is a chronic and rare complication for children who regularly take warfarin after valve surgery of congenital heart disease, which affects their health and quality of life. There are few reports on the rehabilitation nursing experience of children with cerebral infarction after congenital heart valve surgery. Objective: We reported on an 11-year-old child who was diagnosed with cerebral infarction and had undergone rehabilitation care for congenital heart disease and valve replacement. Method: During the period of hospitalization and discharge, rehabilitation care of the children such as functional exercises, language, cognition, daily living ability training and psychological counseling were carried out. Result: In this case, through rehabilitation nursing, the patient's vital signs were stable. His blood pressure fluctuates between 101-124/68-91 mmHg. The weakness of the right limb was relieved compared with the previous one, and the aphasia was improved compared with the previous one. The skin was intact and no bleeding occurred. After 28 days in hospital, the patient was discharged. Conclusion: We summarized the experience of rehabilitation nursing, to provide effective guidance and suggestions for the rehabilitation nursing of children with cerebral infarction after valve replacement.
\end{abstract}

Keywords: Cerebral Infarction, Rehabilitation Nursing, Child, Heart Valve Surgery, Case Report

\section{Introduction}

Stroke refers to persistent neurological deficits caused by acute cerebrovascular diseases [1], including cerebral infarction and cerebral hemorrhage. Studies have found that there are about 70 million stroke patients in China, with more than 2 million new cases per year [2], and about $80 \%-90 \%$ of patients have varying degrees of dysfunction, such as hemiplegia, cognitive impairment and language impairment $[3,4]$. The functional dysfunction of cerebral infarction patients has seriously affected the quality of life of patients, so it is very important to rehabilitate patients. Rehabilitation therapy can make patients return to life smoothly and improve the quality of life. At present, the main incidence of cerebral infarction is the elderly, rarely seen in children. In this case, cerebral infarction occurred during regular warfarin administration after valve replacement for congenital heart disease. Because of his young age, the history of congenital heart disease valve replacement operation and the existing sequelae of cerebral infarction, his rehabilitation and nursing became the key difficulty. We summarizes the experience of rehabilitation nursing, to provide effective guidance and suggestions for the rehabilitation nursing of children with cerebral infarction after valve replacement.

\section{Case Presentation}

An 11-year-old Chinese boy with a history of congenital heart disease valve replacement surgery. Take warfarin 1.875 mg regularly after operation. When the patient got up at 5:00 a.m. on September 30,2020, he had a sudden onset of weakness and dizziness on his right side of the body, and then fell and became unconscious and was sent to the People's Hospital of Suichuan County. On September 30, the CT 
examination of the head considers the left cerebral hemisphere plug. MRI examination showed thickening of the texture of both lungs, right maxillary sinus, bilateral ethmoid sinusitis, and no obvious abnormalities. The head scan on October 27 showed: 1 . Consider the recovery period of cerebral infarction in the parietal insula-basal ganglia area of the left frontotemporal lobe, and leave it with softening and gliosis formation 2. Right basal ganglia softening. So far, the patient has left the right limb with weakness and the right upper limb is heavy. The patient had a limb twitching half a month ago. The patient coughed without drinking water, and water leaked from the corner of his right mouth while drinking. The patient has no dizziness, headache, nausea, vomiting, chest pain, shortness of breath, cough, sputum and other symptoms. The patient now comes to our hospital for further diagnosis and treatment. The outpatient clinic plans to receive "cerebral infarction" into the rehabilitation department for further diagnosis and treatment. Since the onset of illness, the patient has a clear consciousness but cannot answer. In addition, the patient has poor listening comprehension, average orientation and calculation ability, and slightly poor memory. The examination revealed that the patient's left pupil had a diameter of $3 \mathrm{~mm}$ and his right pupil had a diameter of $3 \mathrm{~mm}$, which was sensitive to light reflection and could move freely in all directions. Unfortunately, the patient's left cheek leaked. In addition, the patient's frowning, grinning, and barring teeth cannot be coordinated, the pharyngeal reflex is normal, and the tongue is in the middle. What's more, the patient's right upper limb muscle strength is at the proximal 4 level, and the distal muscle strength is at 3 level. Besides, the patient's right hand cannot perform finger-to-finger, grasping and other actions. Similarly, the patient's right muscle tone increased slightly, the proximal muscle strength of the right lower limb was level 4, and the distal muscle strength was level 2. Fortunately, the muscle strength and muscle tone of the patient's left limb were normal. However, the right foot varus, the finger-nose test and the heel-knee-shin test did not cooperate. Physiological reflexes are normal, but pathological reflexes are not elicited. The patient's spirit, appetite, and sleep are good, and the second stool is normal, and there is no significant weight loss. Laboratory examination was abnormal (Table 1).

Table 1. Abnormal results from laboratory tests.

\begin{tabular}{|c|c|c|c|c|c|}
\hline Item & 11-04 & 10-13 & 11-14 & 11-21 & 11-27 \\
\hline $\begin{array}{l}\text { Novel Coronavirus nucleic RNA } \\
\text { blood routine examination }\end{array}$ & negative & - & - & - & - \\
\hline Mean red blood cell volume (fL) & 81.00 & 81.4 & - & 80.60 & - \\
\hline Absolute value of monocytes $\left({ }^{*} 10^{\wedge} 9 / \mathrm{L}\right)$ & $0.76 \uparrow$ & 0.48 & - & $0.62 \uparrow$ & - \\
\hline Neutrophil absolute value $\left(* 10^{\wedge} 9 / \mathrm{L}\right)$ & $7.07 \uparrow$ & 3.54 & - & 4.09 & - \\
\hline Platelet volume distribution width (fl) & 8.90 & 8.40 & - & 8.90 & - \\
\hline White blood cell count $\left(* 10^{\wedge} 9 / \mathrm{L}\right)$ & $10.35 \uparrow$ & 7.05 & - & 8.03 & - \\
\hline Activated partial thrombin activase time (sec) & $53.9 \uparrow$ & $59.4 \uparrow$ & - & $48.5 \uparrow$ & $47.2 \uparrow$ \\
\hline International standard for prothrombin time & $1.91 \uparrow$ & $2.48 \uparrow$ & - & $1.57 \uparrow$ & 1.29 \\
\hline Prothrombin time $(\mathrm{sec})$ & $22.4 \uparrow$ & $27.8 \uparrow$ & - & $19.0 \uparrow$ & $16.2 \uparrow$ \\
\hline \multicolumn{6}{|l|}{ Serum } \\
\hline Apolipoprotein A (APOA) & $1.78 \uparrow$ & - & $1.66 \uparrow$ & & \\
\hline Lactate dehydrogenase (LDH) & $394 \uparrow$ & - & $405 \uparrow$ & $380 \uparrow$ & $367 \uparrow$ \\
\hline Creatine Kinase (CK) & 153 & - & $1039 \uparrow$ & $279 \uparrow$ & $553 \uparrow$ \\
\hline D-dimer quantification (ng/ml) & 220 & - & - & - & - \\
\hline $\mathrm{N}$-pre-B natriuretic peptide $(\mathrm{pg} / \mathrm{ml})$ & & - & 24 & - & - \\
\hline
\end{tabular}

Written informed consent was obtained from the patient's parents. All procedures performed in studies involving human participants were in accordance with the ethical standards of the institutional and/or national research committee(s) and with the Helsinki Declaration (as revised in 2013).

After the patient was admitted to the hospital, we completed the three major routines, blood biochemistry and other related examinations. In addition, symptomatic treatments such as anticoagulation, lipid-lowering, nourishing nerves, improving circulation, improving cognitive function, and speech function were given. More importantly, rehabilitation treatments such as limb function training for hemiplegia, hand function training, low-frequency pulse electrical stimulation, direct current stimulation, acupuncture and moxibustion treatment, and speech function training were carried out [5].

After heart valve replacement surgery, long-term use of "warfarin" for anticoagulation therapy. After admission, it is necessary to prevent patients from complications such as cardio-cerebral vascular accidents, bleeding, epilepsy, etc. We had a good condition observation including cerebral hemorrhage, gastrointestinal hemorrhage, urinary system hemorrhage, observe the skin hemorrhage of the whole body. And we recorded the patient's 24-hour intake and output, blood pressure changes. The patient had twitches in his hands and feet half a month ago, which may be a "seizure". We closely observed whether the patient had twitches in his hands and feet. Unfortunately, we received the critical value of CK: 1039U/L from the clinical laboratory on November 14, 2020. The medical staff immediately performed electrocardiogram, cardiac color Doppler ultrasound, and special EEG examinations on the patient. And gave the patient low-flow oxygen. We instructed the child to rest, and closely observed whether the child had chest pain, palpitation, palpitations and other uncomfortable symptoms. Additionally, the patient's 
right thumb experienced convulsions and twitches that lasted for 5 minutes. The patient was given $250 \mathrm{mg}$ of sodium valproate sustained-release tablets orally, and fell asleep 3 and a half hours later. There have been no adverse reactions since then.

We gave the patient exercise rehabilitation training including functional exercises, rehabilitation care in different positions, fall prevention, and daily living skills training, because the child had hemiplegia on the right limb or decreased balance ability $[6,7]$. First, exercise the upper limb function: (1) The back of the affected side's hand touches the back of the waist. (2) Bend the shoulders forward $90^{\circ}$, and lift the straight upper limbs forward. (3) In the case of elbow extension, do pronation and supination training for the forearm. (4) When the shoulder is abducted at $90^{\circ}$, the elbow is straightened, and on this basis, the forearm is added for pronation and supination training. (5) Training to consolidate shoulder function. Functional training of the lower limbs: (1) When sitting with the knees bent at $90^{\circ}$, slide the feet backward and dorsiflexion when the heels are grounded. (2) Training of hip extension and knee flexion in the standing position and training of dorsiflexion of the foot when the knee is extended in the standing position [8]. Second, the rehabilitation care of different postures: (1) Placement of the good limbs in the lying position. (2) The good limbs of the sitting position are placed. (3) Posture care during standing training or walking training. Third, prevent falls: Inform the patient's family members of the precautions for activities to prevent the child from falling, including the precautions when getting up, going to the toilet, eating, and changing posture. When children receive various functional exercises, others will help them to avoid falling or accidents. Fourth, daily living ability training: practice washing face, brushing teeth, eating, putting on and taking off clothes, urinating and defecate, posture change, etc $[9,10]$.

In this case, the child with motor aphasia has difficulty in speech expression, and has varying degrees of impairment in retelling, naming, and reading. However, the child can understand simple conversations. Early rehabilitation exercise is helpful to restore their language and cognitive function [11, 12]. Therefore, we conducted cognitive training language rehabilitation training because of cognitive impairment and aphasia. First, the training of comprehension and expression skills: Nursing staff adopt a step-by-step approach and give language guidance [12]. We combine training with daily life, and guide children to practice from simple letters, syllables, graphics, literacy cards, and objects. Second, memory training Inform family members to tell patients what happened in the past and encourage patients to recall past life experiences [11]. Help patients understand the people and things in their current lives to restore memory and reduce misjudgments. Third, psychological care $[13,14]$ : Encourage family members to accompany the patient more, comfort, encourage, support, and enlighten the patient more, including cooperating with rehabilitation treatment, taking the initiative to eat, and walking frequently to maintain a good mood. Fourth, language rehabilitation training: Early use of imitation to train the pronunciation organs, and to instruct patients through oral exhalation for pronunciation training through demonstrative actions [12]. The forms are flexible and diverse, such as allowing children to read pictures, telling stories, training with repeaters, reading aloud and reciting ancient poems, etc., to encourage children to speak more actively [12].

\section{Discussion}

After a stroke, rehabilitation is essential to help survivors reach their optimal level of function and prevent or delay future functional decline [15]. According to the theory of brain plasticity, rehabilitation training patients' external motor and sensory stimulation of the impaired body can restore the lost functions to adapt to the functional changes of the new life and work [16]. Araki $\mathrm{Y}$ et al founded that rehabilitation training can promote transient changes in neural activity during exercise, enhance the activity of the cerebral cortex, increase the thickness of the cerebral cortex and protein content, and promote the formation of new blood vessels [17]. Functional exercise has become the key nursing content for clinical rehabilitation of patients with cerebral infarction after successful rescue.

Nurse strictly check the physician prescribed medication to specification, do a good job in basic nursing, closely monitoring vital signs and condition changes The hospitalization of patients with general evaluation, in the sports evaluation on speech, cognition and ability of daily life in children with disability mainly includes cognitive impairment mood disorders movement dysfunction Speech and language barriers, etc. The training content of the children is mainly sitting balance, standing center of gravity transfer, stepping, eating, changing clothes, excretion, general coordination training, standing balance, practical walking cane use and up and down stairs. In this case, through rehabilitation nursing, the patient's vital signs were stable. His blood pressure fluctuates between 101-124/68-91 mmHg. The weakness of the right limb was relieved compared with the previous one, and the aphasia was improved compared with the previous one. The skin was intact and no bleeding occurred. After 28 days in hospital, the patient was discharged.

This case showed that the rehabilitation and nursing of patients with cerebral infarction plays an important role in the rehabilitation period. By formulating scientific rehabilitation programs and nursing guidance for patients, the course of illness can be shortened, the treatment effect can be improved, and the patient can get rid of the trouble of the disease, and restore health and self-care ability as soon as possible. In addition, in the process of rehabilitation nursing, individualized psychological nursing should be carried out according to the different psychological characteristics and needs of patients. As a meta-analysis suggested, psychological nursing was effective for alleviating depressive symptoms, improving neurological rehabilitation, and recovering the ability of daily life [13]. What's more, for such patients, we need to do a good job of rehabilitation care of hemiplegic limbs in different positions, and combine the 
patients' dysfunctions to provide targeted and personalized functional rehabilitation care. Last but not least, encouraging patients to actively participate can maximize the recovery of patients' motor function, speech function, and cognitive function, and can improve the patient's ability to take care of themselves in life, and help patients return to family and society.

\section{Conclusion}

In conclusion, cerebral infarction is a chronic and rare complication for children who regularly take warfarin after valve surgery of congenital heart disease, which affects their health and quality of life. In addition to the pathogenesis and pathophysiology, there are few reports on the rehabilitation nursing experience of children with cerebral infarction after congenital heart valve surgery. In this case report, we describe the nursing experience of an 11-year-old child with cerebral infarction after congenital heart valve surgery. Good therapeutic effect has been achieved through rehabilitation nursing, such as medication nursing, exercise, speech, cognition, daily life ability training and psychological guidance during hospitalization and discharge. Therefore, we suggested that give the patient exercise rehabilitation training including functional exercises, rehabilitation care in different positions, fall prevention, and daily living skills training to the child who had hemiplegia on the right limb or decreased balance ability.

\section{Conflict of Interest}

All the authors do not have any possible conflicts of interest.

\section{Acknowledgements}

We thank the patient and family who attended this study. Thank all healthcare workers in the Department of Rehabilitation in the First Affiliated Hospital of Jinan University as well as the reviewers and editors.

\section{References}

[1] Feigin V L, Krishnamurthi R V, Parmar P, et al. Update on the Global Burden of Ischemic and Hemorrhagic Stroke in 1990-2013: The GBD 2013 Study [J]. Neuroepidemiology, 2015, 45 (3): 161-176.

[2] Wu S, Wu B, Liu M, et al. Stroke in China: advances and challenges in epidemiology, prevention, and management $[\mathrm{J}]$. The Lancet Neurology, 2019, 18 (4): 394-405.

[3] Nys G M S, Van Zandvoort M J E, De Kort P L M, et al. Cognitive disorders in acute stroke: prevalence and clinical determinants $[\mathrm{J}]$. Cerebrovascular diseases (Basel, Switzerland), 2007, 23 (5-6): 408-416.
[4] Wist S, Clivaz J, Sattelmayer M. Muscle strengthening for hemiparesis after stroke: A meta-analysis [J]. Annals of physical and rehabilitation medicine, 2016, 59 (2): 114-124.

[5] Elsner B, Kugler J, Pohl M, et al. Transcranial direct current stimulation (tDCS) for improving activities of daily living, and physical and cognitive functioning, in people after stroke $[\mathrm{J}]$. The Cochrane database of systematic reviews, 2020, 11 (CD009645).

[6] Wang J, Chen Y, Zhang Y, et al. Rehabilitation nursing for motor functional recovery of acute ischaemic stroke: study protocol for a randomised controlled trial [J]. BMJ open, 2020, 10 (9): e037391.

[7] Chen J, Li S. Clinical Study of Neurology Nursing on Cerebral Apoplexy Rehabilitation [J]. Transl Neurosci, 2019, 10 (164-167).

[8] He C, Xiong C-H, Chen Z-J, et al. Preliminary Assessment of a Postural Synergy-Based Exoskeleton for Post-Stroke Upper Limb Rehabilitation [J]. IEEE transactions on neural systems and rehabilitation engineering: a publication of the IEEE Engineering in Medicine and Biology Society, 2021, 29 (1795-1805).

[9] Yu M, Wang L, Wang H, et al. The effect of early systematic rehabilitation nursing on the quality of life and limb function in elderly patients with stroke sequelae [J]. Am J Transl Res, 2021, 13 (8): 9639-9646.

[10] Tipping C J, Harrold M, Holland A, et al. The effects of active mobilisation and rehabilitation in ICU on mortality and function: a systematic review [J]. Intensive care medicine, 2017, 43 (2): 171-183.

[11] Ye M, Zhao B, Liu Z, et al. Effectiveness of computer-based training on post-stroke cognitive rehabilitation: A systematic review and meta-analysis [J]. Neuropsychol Rehabil, 2020.

[12] Godecke E, Armstrong E A, Rai T, et al. A randomized controlled trial of very early rehabilitation in speech after stroke [J]. International journal of stroke: official journal of the International Stroke Society, 2016, 11 (5): 586-592.

[13] Liao B, Liang M, Ouyang Q, et al. Psychological Nursing of Patients With Stroke in China: A Systematic Review and Meta-Analysis [J]. Frontiers in psychiatry, 2020, 11 (569426).

[14] Farner L, Wagle J, Flekkøy K, et al. Factor analysis of the Montgomery Aasberg Depression Rating Scale in an elderly stroke population [J]. International journal of geriatric psychiatry, 2009, 24 (11): 1209-1216.

[15] Teasell R, Salbach N M, Foley N, et al. Canadian Stroke Best Practice Recommendations: Rehabilitation, Recovery, and Community Participation following Stroke. 6th Edition Update 2019 [J]. International journal of stroke: official journal of the International Stroke Society, 2020, 15 (7): 763-788.

[16] Chen L, Han Z, Gu J. Early Path Nursing on Neurological Function Recovery of Cerebral Infarction [J]. Transl Neurosci, $2019,10(160-163)$.

[17] Araki Y, Furuichi M, Nokura H, et al. [Influence of Pre-existing Cognitive Impairment on Rehabilitation Outcomes in Patients with Cerebral Infarction] [J]. Brain Nerve, 2018, 70 (6): 651-660. 